\title{
Investigating dynamical behaviors of the difference equation
}

$$
x_{n+1}=\frac{C x_{n-5}}{A+B x_{n-2} x_{n-5}}
$$

\section{Ghazel ${ }^{a}$, E. M. Elsayed ${ }^{b, c, *}$, A. E. Matouk ${ }^{a}$, A. M. Mousallam ${ }^{a}$}

${ }^{a}$ Mathematics Department, Faculty of Science, University of Hail, Hail 2440, Saudi Arabia.

${ }^{b}$ Mathematics Department, Faculty of Science, King Abdulaziz University, P. O. Box 80203, Jeddah 21589, Saudi Arabia.

${ }^{c}$ Department of Mathematics, Faculty of Science, Mansoura University, Mansoura 35516, Egypt.

\begin{abstract}
In this work, we investigate the dynamical behaviors of the rational difference equation

$$
x_{n+1}=\frac{C x_{n-5}}{A+B x_{n-2} x_{n-5}}
$$

with arbitrary initial conditions, where $A, B$, and $C$ are arbitrary constants. A general solution is obtained. Asymptotic behavior and asymptotic stability of the equilibrium points are investigated. The existence of the periodic solutions is discussed. Numerical simulations are carried out to verify the analytical results. (c)2017 All rights reserved.
\end{abstract}

Keywords: Rational difference equations, asymptotic behavior, infinite products, local stability, periodicity, convergence. 2010 MSC: 34K13, 34K05, 34K20, 39A10.

\section{Introduction}

Difference equations are used not only to describe dynamical systems that evolves discretely, but also, to present, in the easiest way, many numerical schemes [1-20]. Difference equations have potential applications in various fields of science such as game theory $[2,3,5,6,17,18]$, mathematical biology $[15,34]$, physics, and engineering [11]. Therefore, the study of the qualitative behavior of a nonlinear rational difference equations of higher orders [21-39] is of paramount importance because of its promising applications.

Indeed, some qualitative behaviors of difference equations have been investigated by authors. For examples in [4], Amleh et al. studied the third order rational difference equation

$$
x_{n+1}=\frac{a+b x_{n-1}}{A+B x_{n-2}}
$$

\footnotetext{
${ }^{*}$ Corresponding author

Email addresses: malek_-ghazel@yahoo.fr (M. Ghazel), emmelsayed@yahoo.com (E. M. Elsayed), aematouk@hotmail.com (A. E. Matouk), ahmedmetwally77@hotmail.com (A. M. Mousallam)

doi:10.22436/jnsa.010.09.09
} 
and Elabbasy et al. in [10] studied the global stability character of the following difference equation

$$
x_{n+1}=\frac{C x_{n}+\beta x_{n-1}+\gamma x_{n-2}}{A x_{n}+B x_{n-1}+C x_{n-2}} .
$$

Another approach to investigate a difference equation is to find the analytical formula of the general term. Contrary to the linear case, there is no general method to find such explicit solution. Accordingly, Cinar [8] obtained the solution of the difference equation

$$
x_{n+1}=\frac{a x_{n-1}}{1+b x_{n} x_{n-1}} .
$$

Here, we are concerned with the analytic study of a class of sixth order rational difference equation

$$
x_{n+1}=\frac{C x_{n-5}}{A+B x_{n-2} x_{n-5}},
$$

where $A, B$, and $C$ are arbitrary constants and with arbitrary initial data $x_{-5}=f, x_{-4}=e, x_{-3}=d$, $x_{-2}=c, x_{-1}=b$, and $x_{0}=a$.

Note first that, if $C=0$, then for all $n \in \mathbb{N}, x_{n}=0$, so we will consider that $C \neq 0$. The parameter $C$ is kept in our equation in order to investigate the rich dynamics of the difference equation (1.1) as the variation of this parameter. Also, the cases $A=0$ and $B=0$ are trivial, therefore we will assume that $A \neq 0$ and $B \neq 0$.

Finally, throughout the manuscript, we assume the product $\prod_{p=n}^{m} a_{p}=1$ when $\left(a_{p}\right)_{p}$ is a sequence of complex numbers and $n>m, m, n \in \mathbb{Z}$.

\section{Preliminaries}

A difference equation of order $\mathrm{k}$ is an equation of the form

$$
x_{n+1}=F\left(x_{n}, x_{n-1}, \ldots, x_{n-(k-1)}\right), n=0,1, \ldots,
$$

where $F=F\left(u_{0}, u_{1}, \ldots, u_{k-1}\right)$ is a function that maps on some set $I^{k}$ into I. A solution of Eq. (2.1) is a sequence $x_{n}$ that satisfies Eq. (2.1) for all $n \geqslant 0$. With each solution $x_{n}$ of the Eq. (1.1), we associate the vector of initial conditions $v_{0}(x)=\left(x_{0}, x_{-1}, \ldots, x_{-k+1}\right) \in I^{k}$.

Definition 2.1 (Equilibrium point). A point $\bar{x} \in \mathbb{R}$ is called an equilibrium point of the Eq. (2.1), if

$$
\bar{x}=F(\bar{x}, \bar{x}, \ldots, \bar{x}) .
$$

Let $\bar{x} \in \mathbb{R}$ be an equilibrium point of the Eq. (2.1), and denote by $v(\bar{x}) \in \mathrm{I}^{\mathrm{k}}$ the vector $v(\bar{x})=$ $(\bar{x}, \bar{x}, \ldots, \bar{x})$. Suppose that the function $F$ is continuously differentiable in some open neighborhood of an equilibrium point $\bar{x}$, and consider the linearized equation of (2.1) about the equilibrium point $\bar{x}$ is given as

$$
y_{n+1}=q_{0} y_{n}+q_{1} y_{n-1}+\cdots+q_{k-1} y_{n-(k-1)},
$$

where $q_{i}=\frac{\partial F}{\partial u_{i}}(\bar{x}, \bar{x}, \cdots, \bar{x}), i=0,1, \cdots, k-1$, so the characteristic equation of (2.2) about $\bar{x}$ is defined as

$$
\lambda^{k}-q_{0} \lambda^{k-1}-\cdots-q_{k-2} \lambda-q_{k-1}=0 .
$$

Definition 2.2 ([33]). An equilibrium point of Eq. (2.1) is locally asymptotically stable if all the roots of the characteristic equation (2.3) have absolute value less than one. However, if at least one root of the characteristic equation (2.3) has absolute value greater than one, then this equilibrium point is unstable. 


\section{Definition 2.3.}

1. If no root of the characteristic equation (2.3) has absolute value equal to one, then the equilibrium point $\bar{x}$ of Eq. (2.1) is said to be hyperbolic.

2. If there exists a root of the characteristic equation (2.3) with absolute value equal to one, then the equilibrium point $\bar{x}$ is called nonhyperbolic.

3. An equilibrium point $\bar{x}$ of Eq. (2.1) is said to be saddle if there exist at least two roots of the characteristic equation (2.3) with absolute value above and beyond one.

4. An equilibrium point $\bar{x}$ of Eq. (2.1) is said to be repeller if all roots of the characteristic equation (2.3) have absolute value greater than one.

5. A solution $\left(x_{n}\right)_{n}$ of Eq. (2.1) is said to be periodic with period $p$ or a periodic-p solution if

$$
x_{n+p}=x_{n}, \quad \forall n \geqslant-k .
$$

A solution is called periodic with prime period $p$ if $p$ is the smallest positive integer for which Eq. (2.4) holds.

\section{Analytical expressions of $\left(x_{n}\right)_{n}$}

In the following, we give some analytical expressions of the sequence $\left(x_{n}\right)_{n}$.

Theorem 3.1. Let $\left(x_{n}\right)_{n \geqslant-5}$ be the sequence given by (1.1) and the initial data that follow, then for all $n \geqslant 0$

$$
\begin{aligned}
x_{6 n-5}=\frac{f C^{n} \prod_{p=1}^{n-1}\left(A^{2 p}+B c f \sum_{k=0}^{2 p-1} A^{k} C^{2 p-1-k}\right)}{\prod_{p=0}^{n-1}\left(A^{2 p+1}+B c f \sum_{k=0}^{2 p} A^{k} C^{2 p-k}\right)} \\
x_{6 n-4}=\frac{e C^{n} \prod_{p=1}^{n-1}\left(A^{2 p}+B b e \sum_{k=0}^{2 p-1} A^{k} C^{2 p-1-k}\right)}{\prod_{p=0}^{n-1}\left(A^{2 p+1}+B b e \sum_{k=0}^{2 p} A^{k} C^{2 p-k}\right)} \\
x_{6 n-3}=\frac{d C^{n} \prod_{p=1}^{n-1}\left(A^{2 p}+B a d \sum_{k=0}^{2 p-1} A^{k} C^{2 p-1-k}\right)}{\prod_{p=0}^{n-1}\left(A^{2 p+1}+B a d \sum_{k=0}^{2 p} A^{k} C^{2 p-k}\right)} \\
x_{6 n-1}=b C^{n} \prod_{p=0}^{n-1}\left(\frac{A^{2 p+1}+B b e \sum_{k=0}^{2 p} A^{k} C^{2 p-k}}{x^{2 p+2}+B b e \sum_{k=0}^{2 p+1} A^{k} C^{2 p+1-k}}\right) \\
c C^{n} \prod_{p=0}^{n-1}\left(\frac{A^{2 p+1}+B c f \sum_{k=0}^{2 p} A^{k} C^{2 p-k}}{A^{2 p+2}+B c f \sum_{k=0}^{2 p+1} A^{k} C^{2 p+1-k}},\right.
\end{aligned}
$$




$$
x_{6 n}=a C^{n} \prod_{p=0}^{n-1}\left(\frac{A^{2 p+1}+B a d \sum_{k=0}^{2 p} A^{k} C^{2 p-k}}{A^{2 p+2}+B a d \sum_{k=0}^{2 p+1} A^{k} C^{2 p+1-k}}\right) .
$$

Proof. By induction, we prove the result for $x_{6 n-5}$. For $n=0$, it is easy to verify that

$$
\frac{f C^{0} \prod_{p=1}^{0-1}\left(A^{2 p}+B c f \sum_{k=0}^{2 p-1} A^{k} C^{2 p-1-k}\right)}{\prod_{p=0}^{0-1}\left(A^{2 p+1}+B c f \sum_{k=0}^{2 p} A^{k} C^{2 p-k}\right)}=f=x_{-5} .
$$

Take $n \geqslant 0$ and assume that the results hold for the step $n$, then prove the results for the step $n+1$. We get

$$
x_{6(n+1)-5}=\frac{C x_{6 n-5}}{A+B x_{6 n-2} x_{6 n-5}}=\frac{\frac{f C^{n} \prod_{p=1}^{n-1}\left(A^{2 p}+B c f \sum_{k=0}^{2 p-1} A^{k} C^{2 p-1-k}\right)}{\prod_{p=0}^{n-1}\left(A^{2 p+1}+B c f \sum_{k=0}^{2 p} A^{k} C^{2 p-k}\right)}}{A\left(A^{2 n}+B c f \sum_{k=0}^{2 n-1} A^{k} C^{2 n-1-k}\right)+B c f C^{2 n}} .
$$

Hence, we obtain

$$
x_{6(n+1)-5}=\frac{f C^{n} \prod_{p=1}^{n}\left(A^{2 p}+B c f \sum_{k=0}^{2 p-1} A^{k} C^{2 p-1-k}\right)}{\prod_{p=0}^{n}\left(A^{2 p+1}+B c f \sum_{k=0}^{2 p} A^{k} C^{2 p-k}\right)} .
$$

Similarly, one can easily obtain the other relations. Thus, the proof is completed.

Remark 3.2. According to the expressions (3.1)-(3.6) in the previous theorem, the initial data $a, b, c, d, e$ and $f$ may be chosen to satisfy the condition abcdef $\neq 0$, in order to avoid vanishing one or more of the subsequences of $\left(x_{n}\right)_{n}$ modulo 6 .

Notation 3.3. We denote by $\left(P_{n}\right)_{n}$ the sequence of two variables polynomials defined for every $n \in \mathbb{N}, x$ and $y$ in $\mathbb{R}$ as

$$
P_{n}(x, y)=(A-C+B x y) A^{n}-B x y C^{n} .
$$

The following corollary gives a simplified analytic expression when $A \neq C$.

Corollary 3.4. Consider the sequence $\left(x_{n}\right)_{n}$ defined by the formula (1.1) and the initial data that follow. If $\mathrm{A} \neq \mathrm{C}$, then for all $\mathrm{n} \geqslant 0$

$$
x_{6 n-5}=\frac{f C^{n}(A-C) \prod_{p=1}^{n-1} P_{2 p}(c, f)}{\prod_{p=0}^{n-1} P_{2 p+1}(c, f)}, \quad x_{6 n-4}=\frac{e C^{n}(A-C) \prod_{p=1}^{n-1} P_{2 p}(b, e)}{\prod_{p=0}^{n-1} P_{2 p+1}(b, e)},
$$




$$
\begin{aligned}
x_{6 n-3}=\frac{d C^{n}(A-C) \prod_{p=1}^{n-1} P_{2 p}(a, d)}{\prod_{p=0}^{n-1} P_{2 p+1}(a, d)}, & x_{6 n-2}=c C^{n} \prod_{p=0}^{n-1}\left(\frac{P_{2 p+1}(c, f)}{P_{2 p+2}(c, f)}\right) \\
x_{6 n-1}=b C^{n} \prod_{p=0}^{n-1}\left(\frac{P_{2 p+1}(b, e)}{P_{2 p+2}(b, e)}\right), & x_{6 n}=a C^{n} \prod_{p=0}^{n-1}\left(\frac{P_{2 p+1}(a, d)}{P_{2 p+2}(a, d)}\right) .
\end{aligned}
$$

Proof. It is sufficient to use in the analytical expressions of the subsequences (3.1)-(3.6), the binomial identity

$$
x^{p+1}-y^{p+1}=(x-y) \sum_{k=0}^{p} x^{k} y^{p-k}
$$

where $p$ is a nonnegative integer and $x, y$ are two real numbers. Thus, the proof is obtained directly.

Corollary 3.5. Consider the sequence $\left(x_{n}\right)_{n}$ defined by the formula (1.1) and the initial data that follow. For $\mathrm{A}=\mathrm{C}$, the following subsequences of $\left(\mathrm{x}_{\mathrm{n}}\right)_{\mathrm{n}}$ can be obtained by direct calculations

$$
\begin{aligned}
& x_{6 n-5}=\mathrm{fA} \frac{\prod_{p=1}^{n-1}(A+2 p B c f)}{\prod_{p=0}^{n-1}(A+(2 p+1) B c f)}, \quad x_{6 n-4}=e A \frac{\prod_{p=1}^{n-1}(A+2 p B b e)}{\prod_{p=0}^{n-1}(A+(2 p+1) B b e)}, \\
& x_{6 n-3}=d A \frac{\prod_{p=1}^{n-1}(A+2 p B a d)}{\prod_{p=0}^{n-1}(A+(2 p+1) B a d)}, \\
& x_{6 n-1}=b \prod_{p=0}^{n-1}\left(\frac{A+(2 p+1) B b e}{A+(2 p+2) B b e}\right), \\
& x_{6 n-2}=c \prod_{p=0}^{n-1}\left(\frac{A+(2 p+1) B c f}{A+(2 p+2) B c f}\right), \\
& x_{6 n}=a \prod_{p=0}^{n-1}\left(\frac{A+(2 p+1) B a d}{A+(2 p+2) B b e}\right) .
\end{aligned}
$$

Remark 3.6. A common hypothesis in the study of rational difference equations is the choice of positive coefficients and initial data so that the solution will be automatically well-defined. It is, in general a problem of great difficulty to determine the good set $\mathrm{G}$ of the initial data without finding the analytical expression of the considered sequence. In our study, the good set is determined as follows.

Using Corollaries 3.4 and 3.5, we can obtain easily the good set $G$ of the sequence $\left(x_{n}\right)_{n}$ as follows.

Theorem 3.7. Let $\left(x_{n}\right)_{n}$ be the sequence defined by the formula (1.1) and the initial data that follows. The good set of the sequence $\left(x_{n}\right)_{n}$ is

$$
\mathrm{G}=\left\{(\mathrm{f}, \mathrm{e}, \mathrm{d}, \mathrm{c}, \mathrm{b}, \mathrm{a}) \in \mathbb{R}^{6} \text { such that } \mathrm{ad}, \mathrm{be}, \text { and } \mathrm{cf} \text { belong to } \mathbb{R}-\left\{\frac{-A^{n}}{B \sum_{k=0}^{n-1} A^{k} C^{n-1-k}}, \quad n \in \mathbb{N}\right\}\right\} .
$$

\section{More precisely}

1. when $\mathrm{A} \neq \mathrm{C}$, the good set of the sequence $\left(x_{n}\right)_{n}$ is

$$
G=\left\{(f, e, d, c, b, a) \in \mathbb{R}^{6} \text { such that } a d, b e, \text { and } c f \text { belong to } \mathbb{R}-\left\{\frac{-A^{n}(A-C)}{B\left(A^{n}-C^{n}\right)}, \quad n \in \mathbb{N}\right\}\right\} ;
$$


2. when $\mathrm{A}=\mathrm{C}$, the good set of the sequence $\left(\mathrm{x}_{\mathrm{n}}\right)_{\mathrm{n}}$ is

$$
\mathrm{G}=\left\{(\mathrm{f}, \mathrm{e}, \mathrm{d}, \mathrm{c}, \mathrm{b}, \mathrm{a}) \in \mathbb{R}^{6} \text { such that } \mathrm{cf}, \mathrm{bd} \text {, and ac belong to } \mathbb{R}-\left\{\frac{\mathrm{A}}{\mathrm{BZ}}\right\}\right\} .
$$

The initial conditions will be considered in the corresponding good set.

\section{Stability analysis of the equilibrium points}

Consider the function $\mathrm{F}$ defined on $\mathbb{R}^{6}$ as

$$
F\left(u_{0}, u_{1}, u_{2}, u_{3}, u_{4}, u_{5}\right)=\frac{C u_{5}}{A+B u_{2} u_{5}} .
$$

Using the function F, the Eq. (1.1) can be written as

$$
x_{n+1}=F\left(x_{n}, x_{n-1}, x_{n-2}, x_{n-3}, x_{n-4}, x_{n-5}\right),
$$

which has the following characteristic equation

$$
\lambda^{6}-\mathrm{q}_{0} \lambda^{5}-\cdots-\mathrm{q}_{4} \lambda-\mathrm{q}_{5}=0 .
$$

Theorem 4.1. Let $\left(x_{n}\right)_{n \geqslant-5}$ be a solution of Eq. (1.1) and the initial data that follow.

1. When $\mathrm{B}(\mathrm{A}-\mathrm{C}) \geqslant 0$, the Eq. (1.1) has a unique equilibrium point $\overline{\mathrm{x}}_{1}=0$.

2. When $\mathrm{B}(\mathrm{A}-\mathrm{C})<0$, the Eq. (1.1) has exactly three equilibrium points

$$
\bar{x}_{1}=0, \quad \bar{x}_{2}=\sqrt{\frac{C-A}{B}}, \text { and } \bar{x}_{3}=-\sqrt{\frac{C-A}{B}} .
$$

Theorem 4.2. Let $\left(x_{n}\right)_{n \geqslant-5}$ be a solution of Eq. (1.1) and the initial data that follow.

1. If $\frac{\mathrm{C}}{\mathrm{A}}<0$, then there is no real root for (4.1).

2. If $0 \leqslant \frac{C}{A}<1$, then the equilibrium point $\bar{x}_{1}$ is locally asymptotically stable.

3. If $\mathrm{A}=\mathrm{C}$, then the equilibrium point $\overline{\mathrm{x}}_{1}$ is nonhyperbolic.

4. If $\frac{C}{A}>1$, then the equilibrium point $\bar{x}_{1}$ is a repeller.

Moreover, for $\mathrm{B}(\mathrm{A}-\mathrm{C})<0$,

(i) the equilibrium points $\bar{x}_{2}$ and $\bar{x}_{3}$ are nonhyperbolic;

(ii) if $0<\frac{\mathrm{C}}{\mathrm{A}}<1$, then the equilibrium points $\overline{\mathrm{x}}_{2}$ and $\overline{\mathrm{x}}_{3}$ are unstable.

Proof. First, define $\mathrm{U}=\left(\mathrm{u}_{0}, \mathrm{u}_{1}, \ldots, \mathrm{u}_{5}\right)$ as an arbitrary point in $\mathbb{R}^{6}$ and recall that $\mathrm{q}_{i}=\frac{\partial \mathrm{F}}{\partial u_{i}}(\bar{x}, \bar{x}, \cdots, \bar{x})$ where $\bar{x}$ is an equilibrium point, then we have the following quantities

$$
\frac{\partial F}{\partial u_{2}}(\mathrm{U})=\frac{-C B u_{5}^{2}}{\left(A+B u_{2} u_{5}\right)^{2}}, \frac{\partial F}{\partial u_{5}}(U)=\frac{C A}{\left(A+B u_{2} u_{5}\right)^{2}} .
$$

We also have, for all $U$ in the domain of differentiability of $F$, the following

$$
\frac{\partial F}{\partial u_{0}}(U)=\frac{\partial F}{\partial u_{1}}(U)=\frac{\partial F}{\partial u_{3}}(U)=\frac{\partial F}{\partial u_{4}}(U)=0 \text {. }
$$

For the equilibrium point $\bar{x}_{1}=0$, the quantities $q_{2}$ and $q_{5}$ in Eq. (4.2) are

$$
q_{2}=0 \text { and } q_{5}=\frac{C}{A} \text {. }
$$

The characteristic equation (4.1) of the equilibrium point $\bar{x}_{1}=0$ is

$$
\lambda^{6}-\frac{C}{A}=0
$$

Thus, we have the following cases. 
1. If $\frac{C}{A}<0$, then Eq. (4.3) has no real root.

2. If $0<\frac{C}{A}<1$, then the real roots are $\pm\left(\frac{C}{A}\right)^{\frac{1}{6}}$. So all the roots of Eq. (4.3) have absolute value less than one which imply that the equilibrium point $\bar{x}_{1}$ is locally asymptotically stable.

3. If $C=A$, then the real roots of Eq. (4.3) are -1 and 1 , so $\bar{x}_{1}$ is nonhyperbolic.

4. If $\frac{C}{A}>1$, then all the roots of Eq. (4.3) have absolute value greater than one, so $\bar{x}_{1}$ is a repeller.

However, when $B(A-C)<0$, the two equilibrium points $\bar{x}_{2}$ and $\bar{x}_{3}$ appear. According to Eq. (4.2), their quantities $q_{2}$ and $q_{5}$ are the same and they are given as follows

$$
q_{2}=\frac{\partial F}{\partial u_{2}}\left(\bar{X}_{i}\right)=\frac{A-C}{C} \text { and } q_{5}=\frac{\partial F}{\partial u_{5}}\left(\bar{X}_{i}\right)=\frac{A}{C^{\prime}}
$$

where $\bar{x}_{i}=\left(\bar{x}_{i}, \bar{x}_{i}, \bar{x}_{i}, \bar{x}_{i}, \bar{x}_{i}, \bar{x}_{i}\right), i=2,3$. Thus, the equilibrium points $\bar{x}_{2}$ and $\bar{x}_{3}$ have the same characteristic equation which is given by

$$
\lambda^{6}-\frac{A-C}{C} \lambda^{3}-\frac{A}{C}=0 .
$$

The real roots of Eq. (4.4) are, -1 and $\left(\frac{A}{C}\right)^{\frac{1}{3}}$, then the equilibrium points $\bar{x}_{2}$ and $\bar{x}_{3}$ of $(1.1)$ are nonhyperbolic. Furthermore, if $\frac{A}{C}>1$, then Eq. (4.4) has at least one root with absolute value greater than one which implies that $\bar{x}_{2}$ and $\bar{x}_{3}$ are unstable.

\section{Convergence}

In this section, we study the convergence of the solution of difference equation (1.1).

5.1. The case $\left|\frac{\mathrm{A}}{\mathrm{C}}\right|<1$.

Theorem 5.1. Let $\left(x_{n}\right)_{n \geqslant-5}$ be the sequence given by the formula (1.1) and the initial data that follow. Assume that $\left|\frac{\mathrm{A}}{\mathrm{C}}\right|<1$, then the subsequences $\left(\mathrm{x}_{6 \mathrm{n}-\mathrm{j}}\right)_{n}, \mathrm{j} \in\{0,1, \ldots, 5\}$ defined in (3.1)-(3.6) converge.

Proof. We distinguish the following two cases.

1. First case, when $A-C+B c f \neq 0$, so by Corollary 3.4, we get

$$
\begin{aligned}
& x_{6 n-5}=(A-C) f C^{n} \frac{\prod_{p=1}^{n-1}\left((A-C+B c f) A^{2 p}+B c f C^{2 p}\right)}{\prod_{p=0}^{n-1}\left((A-C+B c f) A^{2 p+1}+B c f C^{2 p+1}\right)} \\
& =\frac{(A-C) f C^{n}}{(A-C+B c f) A+B c f C} \prod_{p=1}^{n-1}\left(\frac{B c f C^{2 p}\left(\frac{(A-C+B c f)}{B c f}\left(\frac{A}{C}\right)^{2 p}+1\right)}{B c f C^{2 p+1}\left(\frac{(A-C+B c f)}{B c f}\left(\frac{A}{C}\right)^{2 p+1}+1\right)}\right) \\
& =\frac{f C}{A+B c f} \prod_{p=1}^{n-1}\left(\frac{1+\frac{A-C+B c f}{B c f}\left(\frac{A}{C}\right)^{2 p}}{1+\frac{(A-C+B c f)}{B c f}\left(\frac{A}{C}\right)^{2 p+1}}\right)=\frac{f C}{A+B c f} \prod_{p=1}^{n-1} u_{p},
\end{aligned}
$$

where $\left(U_{p}\right)_{p}$ is the sequence defined as $U_{p}=\frac{1+\alpha\left(\frac{A}{C}\right)^{2 p}}{1+\alpha\left(\frac{A}{C}\right)^{2 p+1}}$ and $\alpha=\frac{A-C+B c f}{B c f}$.

For $p$ big enough, $U_{p}$ is always greater than one or lies between zero and one. Consequently, Taylor expansion can be applied for $\mathrm{U}_{\mathrm{p}}$ as follows

$$
\mathrm{u}_{\mathrm{p}}=1+\alpha\left(\frac{\mathrm{A}}{\mathrm{C}}\right)^{2 \mathrm{p}}\left(1-\frac{\mathrm{A}}{\mathrm{C}}\right)+\mathrm{o}\left(\left(\frac{\mathrm{A}}{\mathrm{C}}\right)^{2 \mathrm{p}}\right),
$$

which implies that $U_{p}$ is equivalent to $1+\alpha\left(\frac{A}{C}\right)^{2 p}\left(1-\frac{A}{C}\right)$, where this quantity represents the general term of convergent infinite product. Therefore, the subsequence $\left(x_{6 n-5}\right)_{n}$ is convergent. 
2. In the second case where $A-C+B c f=0$, the subsequence $\left(x_{6 n-5}\right)_{n}$ is constant and equals $f$. Hence, it is convergent. Similarly, the proof for the other subsequences $\left(x_{6 n-j}\right)_{n}, j=0, \ldots, 4$ can be obtained. Thus, the proof is completed.

Remark 5.2 (Commentary on the convergence of $\left(x_{n}\right)_{n}$ in the case $\left|\frac{A}{C}\right|<1$ ). We have proved that, in the case when $\left|\frac{A}{C}\right|<1$, every subsequence $\left(x_{6 n-j}\right)_{n}(j=0,1,2,3,4,5)$ converges to a real number $l_{j}$. So a question arises about the convergence of the whole sequence $\left(x_{n}\right)_{n}$. To answer it, we first consider the subsequences $\left(x_{6 n-1}\right)_{n}$ and $\left(x_{6 n-4}\right)_{n}$ which are related by the equations

$$
\begin{aligned}
& x_{6(n+1)-1}=\frac{C x_{6 n-1}}{A+B x_{6(n+1)-4} x_{6 n-1}}, \\
& x_{6(n+1)-4}=\frac{C x_{6 n-4}}{A+B x_{6 n-1} x_{6 n-4}} .
\end{aligned}
$$

Taking the limit on $n$ in the Eq. (5.1), we obtain $l_{1}=\frac{\mathrm{Cl}_{1}}{\mathrm{~A}+\mathrm{Bl} l_{1} l_{4}}$, which is equivalent to

$$
S_{1}:\left\{\begin{array}{l}
l_{1}=0, \\
\text { or } \\
l_{1} \neq 0 \text { and } l_{4}=\frac{C-A}{B l_{1}} .
\end{array}\right.
$$

Also, taking the limit on $n$ in the Eq. (5.2), we obtain $l_{4}=\frac{\mathrm{Cl}_{4}}{\mathrm{~A}+\mathrm{Bl}_{4} \mathrm{l}_{1}}$, which is equivalent to

$$
S_{2}:\left\{\begin{array}{l}
l_{4}=0 \\
\text { or } \\
l_{1} \neq 0 \text { and } l_{4}=\frac{C-A}{\mathrm{Bl}_{1}} .
\end{array}\right.
$$

Combining the systems $S_{1}, S_{2}$ and since $A \neq C$, we obtain

$$
S:\left\{\begin{array}{l}
l_{1}=l_{4}=0, \\
\text { or } \\
l_{1} \neq 0 \text { and } l_{4}=\frac{C-A}{B l_{1}} .
\end{array}\right.
$$

However, the proposition $l_{1}=l_{4}=0$ contradicts the fact that the infinite product $\Pi_{p} \geqslant 0 u_{p}$ converges, hence the only possibility is that

$$
l_{1} \neq 0, l_{4} \neq 0, \text { and } l_{4}=\frac{C-A}{B l_{1}} .
$$

Then, denote by $g$ the function defined on $\mathbb{R}-\{0\}$ as $g(x)=\frac{C-A}{B x}$, where

$$
g(x)=x \text { iff } x^{2}=\frac{C-A}{B} .
$$

Hence, $g$ has fixed points in $\mathbb{R}-\{0\}$ iff $\frac{C-A}{B}>0$, which are given as $\mp \sqrt{\frac{C-A}{B}}$. It can be similarly shown that

(i) the limits $l_{2}$ and $l_{5}$ are associated to each other under the mapping $g$,

(ii) the limits $l_{3}$ and $l_{6}$ are associated to each other under the mapping $g$.

The numerical example (see Fig. 1 ) illustrates that, $l_{1}$ and $l_{4}$ are not necessarily the same even we choose $\frac{C-A}{B}>0$ and $\left|\frac{A}{C}\right|<1$, which implies that the sequence $\left(x_{n}\right)_{n}$ may converge or diverge. 


\subsection{The case $\mathrm{C}=\mathrm{A}$.}

Theorem 5.3. Let $\left(x_{n}\right)_{n \geqslant-5}$ be the sequence given by the formula (1.1) and the initial data that follow. If $A=C$, then the sequence $\left(x_{n}\right)_{n}$ converges toward zero.

Proof. Replacing C with A into Corollary 3.5, we get

$$
x_{6 n-5}=\frac{f A}{A+B c f} \prod_{p=1}^{n-1}\left(\frac{A+2 p B c f}{A+(2 p+1) B c f}\right)=\frac{f A}{A+B c f} \prod_{p=1}^{n-1} v_{p}
$$

where $V_{p}=\frac{1+\frac{A}{2 p B c f}}{1+\frac{A}{2 p B c f}+\frac{1}{2 p}}$. Furthermore, there exists $p_{0} \in \mathbb{N}$ such that, for all $p \geqslant p_{0}, V_{p} \in(0,1)$. Then, the Taylor expansion gives

$$
V_{p}=\left(1+\frac{1}{2 p B c f}\right)\left(1-\frac{1+B c f}{2 p B c f}+o\left(\frac{1}{p}\right)\right)=1-\frac{1}{2 p}+o\left(\frac{1}{p}\right),
$$

which is the general term of divergent infinite product. Since for $p$ big enough, $0<V_{p}<1$, then $\lim _{n \rightarrow \infty} \prod_{p=1}^{n-1} u_{p}=0$, which implies that the subsequence $\left(x_{6 n-5}\right)_{n}$ converges to zero. Similarly, one can easily prove that the other subsequences converge to zero, therefore the sequence $\left(x_{n}\right)_{n}$ converges to zero which completes the proof.

\subsection{The case $\mathrm{C}=-\mathrm{A}$.}

Theorem 5.4. Let $\left(x_{n}\right)_{n \geqslant-5}$ be the sequence given by the formula (1.1) and the initial data that follow, and suppose that $\mathrm{AB}<0$, if $\mathrm{C}=-\mathrm{A}$, we have the following propositions:

1. the subsequence $\left(x_{6 n-5}\right)_{n}$ converges iff cf $\in(-\infty, 0) \cup\left[\frac{-2 A}{B}, \infty\right)$. However, the subsequence $\left(x_{6 n-2}\right)_{n}$ converges iff $\mathrm{cf} \in\left(0, \frac{-2 \mathrm{~A}}{\mathrm{~B}}\right]$;

2. the subsequence $\left(\mathrm{x}_{6 \mathrm{n}-4}\right)_{\mathrm{n}}$ converges iff be $\in(-\infty, 0) \cup\left[\frac{-2 \mathrm{~A}}{\mathrm{~B}}, \infty\right)$. However, the subsequence $\left(\mathrm{x}_{6 \mathrm{n}-1}\right)_{\mathrm{n}}$ converges iff be $\in\left(0, \frac{-2 \mathrm{~A}}{\mathrm{~B}}\right]$;

3. the subsequence $\left(x_{6 n}-3\right)_{n}$ converges iff ad $\in(-\infty, 0) \cup\left[\frac{-2 A}{B}, \infty\right)$. However, the subsequence $\left(x_{6 n}\right)_{n}$ converges iff $\mathrm{ad} \in\left(0, \frac{-2 A}{B}\right]$.

Proof.

1. (a) Replacing $C$ by $(-A)$ in Corollary 3.4 for the subsequence $\left(x_{6 n-5}\right)_{n}$, we obtain

$$
\begin{aligned}
x_{6 n-5}=f(-A)^{n} 2 A & \frac{\prod_{p=1}^{n-1}\left((2 A+B c f) A^{2 p}+B c f(-A)^{2 p}\right)}{\prod_{p=0}^{n-1}\left((2 A+B c f) A^{2 p+1}+B c f(-A)^{2 p+1}\right)} \\
=\frac{f(-A)^{n}}{A+B c f} & \prod_{p=1}^{n-1}\left(\frac{2 A^{2 p+1}}{2 A^{2 p+2}+2 B c f A^{2 p+1}}\right)=f\left(\frac{-1}{1+\frac{B c f}{A}}\right)^{n} .
\end{aligned}
$$

Thus, $\left(x_{6 n-5}\right)_{n}$ converges iff $\left\{\begin{array}{l}\left|1+\frac{B c f}{A}\right|>1, \\ \text { or } 1+\frac{B c f}{A}=-1\end{array}\right.$ This is equivalent to $c f \in(-\infty, 0) \cup\left[\frac{-2 A}{B}, \infty\right)$. So, we deduce that 
(i) $c f \in(-\infty, 0) \cup\left(\frac{-2 A}{B}, \infty\right)$ is equivalent to $\left(x_{6 n}-5\right)_{n}$ converges to zero,

(ii) $c f \in\left(0, \frac{-2 A}{B}\right)$ is equivalent to $\left(x_{6 n-5}\right)_{n}$ goes to infinity,

(iii) $c f=\frac{-2 A}{B}$ is equivalent to $x_{6 n-5}=f$.

(b) To prove the other part of $(1)$, we replace $C$ by $(-A)$ in Corollary 3.4 for the subsequence $\left(x_{6 n-2}\right)_{n}$, then we get

$$
x_{6 n-2}=c(-A)^{n} \prod_{p=0}^{n-1}\left(\frac{A^{2 p+1}+B c f A^{2 p}}{A^{2 p+2}}\right)=c\left(-\left(1+\frac{B c f}{A}\right)\right)^{n}=\frac{c f}{x_{6 n-5}} .
$$

Consequently, for all $c$ and $f$ in $\mathbb{R}$ such that $c f \neq \frac{-2 A}{B}$, the subsequence $\left(x_{6 n-2}\right)_{n}$ converges iff $\left(x_{6 n-5}\right)_{n}$ goes to a nonzero limit, which is equivalent (by the assertions (i) and (ii)) that $\left(\left|x_{6 n}-5\right|\right)_{n}$ diverges to infinity. The last assertion holds if and only if $\mathrm{cf} \in\left(0, \frac{-2 A}{B}\right)$.

Similar relations hold between the subsequences $\left(x_{6 n-4}\right)_{n},\left(x_{6 n-1}\right)_{n}$, and between the subsequences $\left(x_{6 n-3}\right)_{n},\left(x_{6 n}\right)_{n}$ for proving (2) and (3). This completes the proof.

\section{Remark 5.5.}

(i) The case $C=-A$ and $A B>0$ give a quite similar results.

(ii) It is easy to see that, for $C=-A$, the only possibility for $\left(x_{n}\right)_{n}$ to be convergent is when $a=b=$ $c=d=e=f= \pm \sqrt{\frac{-2 A}{B}}$ and $\left(x_{n}\right)_{n}$ is equal to this common value of initial data, i.e., $x_{n}=a$.

5.4. The case $\left|\frac{\mathrm{A}}{\mathrm{C}}\right|>1$.

Theorem 5.6. Let $\left(x_{n}\right)_{n \geqslant-5}$ be the sequence given by the formula (1.1) and the initial data that follow. Assume that $\left|\frac{\mathrm{A}}{\mathrm{C}}\right|>1$, then

1. for all $j \in\{0, \ldots, 5\}$, the subsequence $\left(x_{6 n-j}\right)_{n}$ converges;

2. the sequence $\left(x_{n}\right)_{n}$ converges iff $(A-C+B c f)(A-C+B b e)(A-C+B a d) \neq 0$, or $A-C+B c f=$ $\mathrm{A}-\mathrm{C}+\mathrm{Bbe}=\mathrm{A}-\mathrm{C}+\mathrm{Bad}=0$ and $\mathrm{a}=\mathrm{b}=\mathrm{c}=\mathrm{d}=\mathrm{e}=\mathrm{f}$.

Proof.

1. For the subsequence $\left(x_{6 n-5}\right)$, we distinguish two cases.

(a) For $A-C+B c f \neq 0$, according to Corollary 3.4

$$
\begin{aligned}
x_{6 n-5}=(A-C) f C^{n} \frac{\prod_{p=1}^{n-1}\left((A-C+B c f) A^{2 p}+B c f C^{2 p}\right)}{\prod_{p=0}^{n-1}\left((A-C+B c f) A^{2 p+1}+B c f C^{2 p+1}\right)} \\
=\frac{f C}{A+B c f}\left(\frac{C}{A}\right)^{n-1} \prod_{p=1}^{n-1}\left(\frac{1+\frac{B c f}{A-C+B c f}\left(\frac{C}{A}\right)^{2 p}}{1+\frac{B c f}{A-C+B c f}\left(\frac{C}{A}\right)^{2 p+1}}\right)=\frac{f C}{A+B c f}\left(\frac{C}{A}\right)^{n-1} \prod_{p=1}^{n-1} W_{p},
\end{aligned}
$$

where $\left(W_{p}\right)_{p}$ is the sequence defined as $W_{p}=\frac{1+\beta\left(\frac{c}{A}\right)^{2 p}}{1+\beta\left(\frac{c}{A}\right)^{2 p+1}}$ and $\beta=\frac{B c f}{A-C+B c f}$. Also, there exists $p_{0} \in \mathbb{N}$ such that either for all $p \geqslant p_{0}, W_{p} \in(0,1)$ or for all $p \geqslant p_{0}, W_{p}>1$. Hence, the Taylor expansion gives

$$
W_{p}=1+\beta\left(1-\frac{C}{A}\right)\left(\frac{C}{A}\right)^{2 p}+o\left(\left(\frac{C}{A}\right)^{2 p+1}\right),
$$

which is the general term of convergent infinite product, thus $\left(x_{6 n}-5\right)_{n}$ converges to zero. Similarly, one can prove that the subsequence $\left(x_{6 n-2}\right)_{n}$ converges to zero and that, 
(i) for $A-C+B b e \neq 0$, the subsequences $\left(x_{6 n-4}\right)_{n}$ and $\left(x_{6 n-1}\right)_{n}$ converge to zero;

(ii) for $A-C+B a d \neq 0$, the subsequences $\left(x_{6 n}-3\right)_{n}$ and $\left(x_{6 n}\right)_{n}$ converge to zero.

(b) For $A-C+B c f=0$, by Corollary 3.4, the subsequences $\left(x_{6 n-5}\right)_{n}$ and $\left(x_{6 n-2}\right)_{n}$ are constants, $x_{6 n-5}=$ $f$ and $x_{6 n-2}=c$.

Similarly, by Corollary 3.4,

(i) for $A-C+B b e=0$, the subsequences $\left(x_{6 n-4}\right)_{n}$ and $\left(x_{6 n-1}\right)_{n}$ are constants, $x_{6 n-4}=e$ and $x_{6 n-1}=b$

(ii) for $A-C+B a d=0$, the subsequences $\left(x_{6 n-3}\right)_{n}$ and $\left(x_{6 n}\right)_{n}$ are constants, $x_{6 n-3}=d$ and $x_{6 n}=a$.

2. The preview calculus shows that

(a) if $(A-C+B c f)(A-C+B b e)(A-C+B a d) \neq 0$, then the sequence $\left(x_{n}\right)_{n}$ converges to zero;

(b) if $A-C+B c f=A-C+B b e=A-C+B a d=0$, then the subsequences $\left(x_{6 n-j}\right)_{n}, j=0,1, \ldots, 5$ are constants: $x_{6 n-5}=f, x_{6 n-4}=e, x_{6 n-3}=d, x_{6 n-2}=c, x_{6 n-1}=b$ and $x_{6 n}=a$, then $\left(x_{n}\right)_{n}$ converges if and only if $a=b=c=d=e=f$;

(c) consider for instance the case $A-C+B c f=0$ and $A-C+B b e \neq 0$, the preview computations show that the subsequences $\left(x_{6 n-5}\right)_{n}$ and $\left(x_{6 n-2}\right)_{n}$ are constants, $x_{6 n-5}=f$ and $x_{6 n-2}=c$, and that the subsequences $\left(x_{6 n-5}\right)_{n},\left(x_{6 n-2}\right)_{n}$ converge to zero, so if $\left(x_{n}\right)_{n}$ converges, then $f=c=0$, this implies that $A=C$ (since $A-C+B c f=0$ ), which contradicts the fact that $\left|\frac{A}{C}\right|>1$. Thus $\left(x_{n}\right)_{n}$ diverges and this achieves the proof.

Based on the calculus in all preview cases, if $C=-A$ and abcdef $\neq\left(\frac{-2 A}{B}\right)^{3}$, then one of the products of $c f, e b$, and $d a$ is different of $\frac{-2 A}{B}$, so the sequence $\left(x_{n}\right)_{n}$ is not bounded. Therefore, the following theorem is now proved.

Theorem 5.7 (Boundedness of $\left(x_{n}\right)_{n}$ ). The difference equation (1.1) has an unbounded solution iff $C=-\mathrm{A}$ and abcdef $\neq\left(\frac{-2 A}{B}\right)^{3}$.

\section{Periodicity}

In the sequel, we need the following lemma, which describes sufficient conditions for Eq. (1.1) to have a periodic solution.

Lemma 6.1. Let $\left(x_{n}\right)_{n \geqslant-5}$ be a solution of Eq. (1.1) and the initial data that follow. Suppose that there are real numbers $l_{5}, l_{4}, l_{3}, l_{2}, l_{1}, l_{0}$ such that $\lim _{n \rightarrow \infty} x_{6 n-j}=l_{j}$ for $j=0, \ldots, 5$. Then let $\left(y_{n}\right)_{n \geqslant-5}$ be the period-6 sequence such that

$$
y_{-j}=l_{j} \text { for all } j=0, \ldots, 5 \text {. }
$$

Consequently, the sequence $\left(y_{n}\right)_{n \geqslant-5}$ is a period-6 solution of Eq. (1.1).

The periodicity results are given by the following theorem.

Theorem 6.2. Let $\left(x_{n}\right)_{n \geqslant-5}$ be a solution of Eq. (1.1) and the initial data that follow, then

1. for $\left|\frac{\mathrm{A}}{\mathrm{C}}\right|<1$, Eq. (1.1) has a periodic- 6 solution;

2. for $\mathrm{C}=-\mathrm{A}, \mathrm{Eq}$. (1.1) has a periodic-6 solution iff $\mathrm{cf}=\mathrm{be}=\mathrm{ad}=\frac{-2 \mathrm{~A}}{\mathrm{~B}}$;

3. for $\left|\frac{A}{C}\right|>1$, if $(A-C+B c f)(A-C+B b e)(A-C+B a d)=0$, then Eq. (1.1) has a nontrivial periodic- 6 solution, more precisely,

(a) if $\mathrm{A}-\mathrm{C}+\mathrm{Bcf}=\mathrm{A}-\mathrm{C}+\mathrm{Bbe}=\mathrm{A}-\mathrm{C}+\mathrm{Bad}=0$, then the solution of $\mathrm{Eq}$. (1.1) is a periodic- 6 solution;

(b) if at least one but not all the following terms $\mathrm{A}-\mathrm{C}+\mathrm{Bcf}, \mathrm{A}-\mathrm{C}+\mathrm{Bbe}$ and $\mathrm{A}-\mathrm{C}+\mathrm{Bad}$ is equal to zero, then Eq. (1.1) has a periodic-6 solution. 
Proof.

1. Suppose that $\left|\frac{A}{C}\right|<1$, then by Theorem 5.1, there are real numbers $l_{5}, l_{4}, l_{3}, l_{2}, l_{1}$, and $l_{0}$, such that $\lim _{n \rightarrow \infty} x_{6 n-j}=l_{j}$ for all $j=0, \ldots, 5$. Applying Lemma 6.1, it follows that the sequence

$$
l_{5}, l_{4}, l_{3}, l_{2}, l_{1}, l_{0}, l_{5}, l_{4}, l_{3}, l_{2}, l_{1}, l_{0}, \ldots,
$$

is a periodic-6 solution of Eq. (1.1).

2. In the case of $C=-A$, if $A B<0$, then using Theorems 5.4 and 5.7, we find that at $c f=b e=a d=$ $\frac{-2 A}{B}$, Eq. (1.1) has a periodic- 6 solution

$$
f, e, d, c, b, a, f, e, d, c, b, a, \ldots .
$$

If abcdef $\neq\left(\frac{-2 A}{B}\right)^{3}$, the solution of Eq. (1.1) is unbounded therefore it has no periodic solutions. The same results hold when $A B>0$.

3. In the case of $\left|\frac{A}{C}\right|>1$, we consider the following.

(a) If $A-C+B c f=A-C+B b e=A-C+B a d=0$, then by (2) (b) in the proof of Theorem 5.6 , the subsequences $\left(x_{6 n-j}\right)_{n}, j=0,1, \ldots, 5$ are constants: $x_{6 n-5}=f, x_{6 n-4}=e, x_{6 n-3}=d$, $x_{6 n-2}=c, x_{6 n-1}=b$ and $x_{6 n}=a$, so the solution of Eq. (1.1) is a periodic- 6 solution

$$
f, e, d, c, b, a, f, e, d, c, b, a, \ldots \text {. }
$$

(b) Suppose for instance that $A-C+B c f=A-C+B b e=0$ and $A-C+B a d \neq 0$, then by (1) (a) and (1) (b) in the proof of Theorem 5.6, we get $x_{6 n-5}=f, x_{6 n-4}=e, x_{6 n-2}=c, x_{6 n-1}=b$, and the subsequences $\left(x_{6 n-3}\right)_{n},\left(x_{6 n}\right)_{n}$ converge to zero. Applying Lemma 6.1, we obtain the following periodic-6 solution of Eq. (1.1)

$$
f, e, 0, c, b, 0, f, e, 0, c, b, 0, \ldots \text {. }
$$

This completes the proof.

\section{Numerical simulations}

Even if it will be possible to obtain analytical conditions, it would be quite difficult to deal with them. So, in order to analyze the dynamical behaviors of Eq. (1.1), we investigate the general solutions of the whole sequence and subsequences of the proposed equation, by using numerical simulation. Therefore we study the following cases numerically.

1. The case $\left|\frac{A}{C}\right|<1$ can be obtained by choosing the parameter values $A=0.5, B=C=1$ and the initial data $a=-1, b=0.5, c=-0.2, d=0.8, e=0.5, f=0.4$. In Fig. 1 , the simulation results show that the subsequences $\left(x_{6 n-j}\right)_{n}, j=0,1, \ldots, 5$ converge which match Theorem 5.1 and the whole sequence $\left(x_{n}\right)_{n}$ diverges which matches Remark 5.2.

2. The case $C=A$ is investigated using the parameter values $A=C=0.5, B=1$ and the initial data $a=0.1, b=0.2, c=0.3, d=-0.4, e=-0.5, f=1$. The solution of Eq. (1.1) converges to zero is depicted in Fig. 2 which is coherent to Theorem 5.3.

3. The case $C=-A, A B<0$, cf $\in(-\infty, 0) \cup\left(\frac{-2 A}{B}, \infty\right)$, be $\in\left(0, \frac{-2 A}{B}\right)$ and $a d \in(-\infty, 0) \cup\left(\frac{-2 A}{B}, \infty\right)$ is discussed using the parameter values $A=2, B=-4, C=-2$ and the initial data $a=0.2, b=0.3$, $c=0.1, d=-0.3, e=0.5, f=-1$. In Fig. 3 , it is shown that the subsequences $\left(x_{6 n-5}\right)_{n},\left(x_{6 n-1}\right)_{n}$, $\left(x_{6 n-3}\right)_{n}$ converge to zero, however the subsequences $\left(\left|x_{6 n-2}\right|\right)_{n},\left(\left|x_{6 n-4}\right|\right)_{n},\left(\left|x_{6 n}\right|\right)_{n}$ go to infinity which are coherent to Theorem 5.4. Hence, the solution is unbounded which matches Theorem 5.7. 

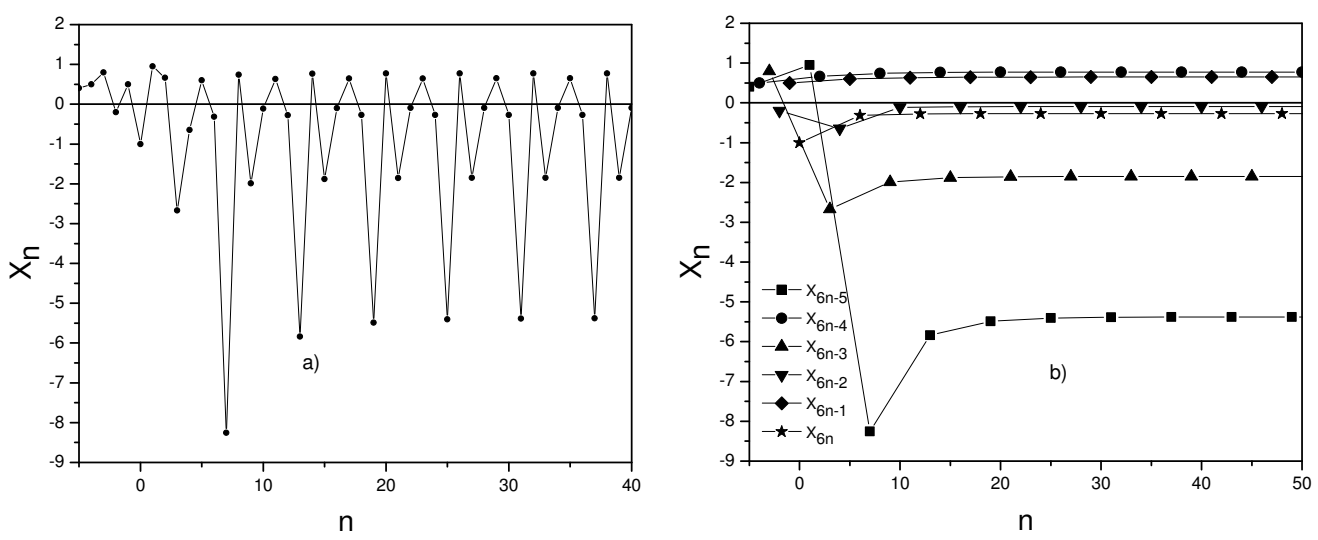

Figure 1: Using the parameter values $A=0.5, B=C=1$ and the initial data $a=-1, b=0.5, c=-0.2, d=0.8, e=0.5, f=0.4$; (a) the sequence $\left(x_{n}\right)_{n}$ diverges, $(b)$ the subsequences $\left(x_{6 n-j}\right)_{n}, j=0,1, \ldots, 5$ converge.
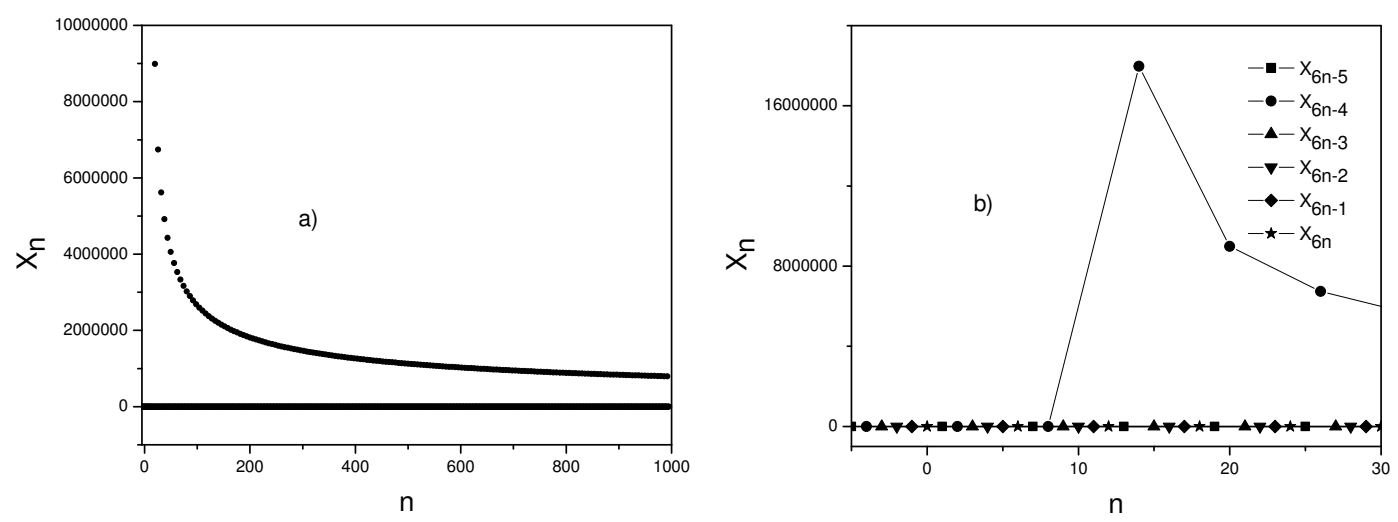

Figure 2: Using the parameter values $A=C=0.5, B=1$ and the initial data $a=0.1, b=0.2, c=0.3, d=-0.4, e=-0.5, f=1$; (a) the whole sequence $\left(x_{n}\right)_{n}$ converges to zero, (b) the corresponding subsequences are illustrated.
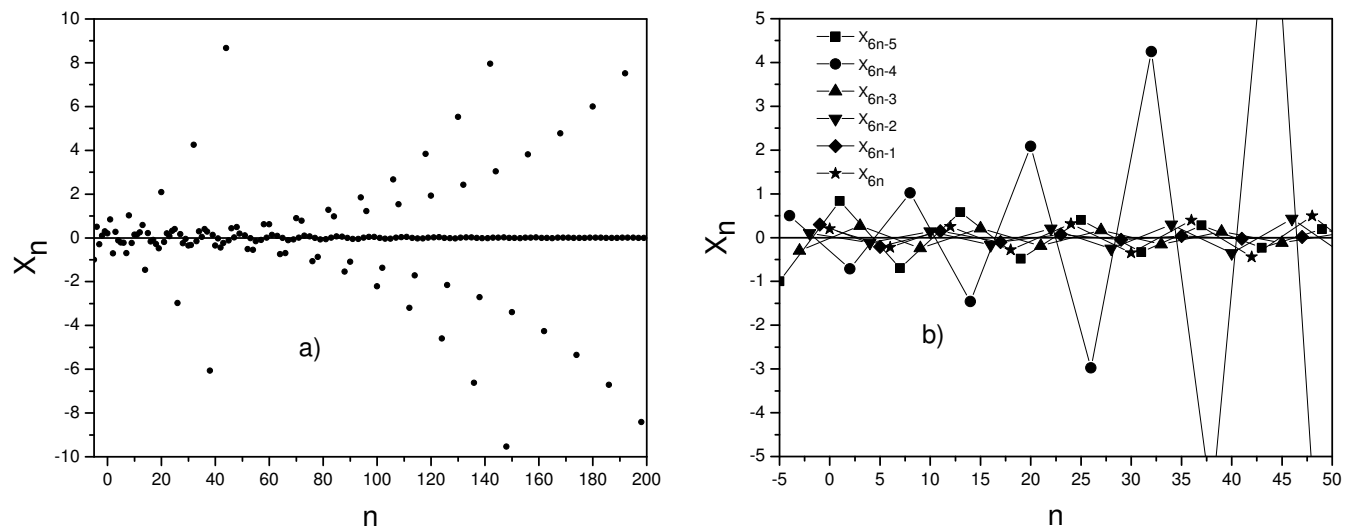

Figure 3: Using the parameter values $A=2, B=-4, C=-2$ and the initial data $a=0.2, b=0.3, c=0.1, d=-0.3, e=0.5$, $f=-1$; (a) the whole sequence is unbounded, (b) the corresponding subsequences are illustrated. 
4. The case $C=-A, A B<0, c f=\frac{-2 A}{B}$, be $\in\left(0, \frac{-2 A}{B}\right)$ and $a d \in\left(0, \frac{-2 A}{B}\right)$ is studied using the parameter values $A=2, B=-4, C=-2$ and the initial data $a=0.2, b=0.3, c=\frac{1}{3}, d=0.3, e=0.5, f=3$. In Fig. 4, it is shown that the subsequences $\left(x_{6 n-5}\right)_{n}$ and $\left(x_{6 n-2}\right)_{n}$ are constants, $x_{6 n-5}=f, x_{6 n-2}=c$, $\left(x_{6 n-1}\right)_{n}$ and $\left(x_{6 n}\right)_{n}$ converge to zero, however the subsequences $\left(\left|x_{6 n-3}\right|\right)_{n},\left(\left|x_{6 n-4}\right|\right)_{n}$ go to infinity which match Theorem 5.4. Therefore the solution is unbounded which agrees Theorem 5.7.
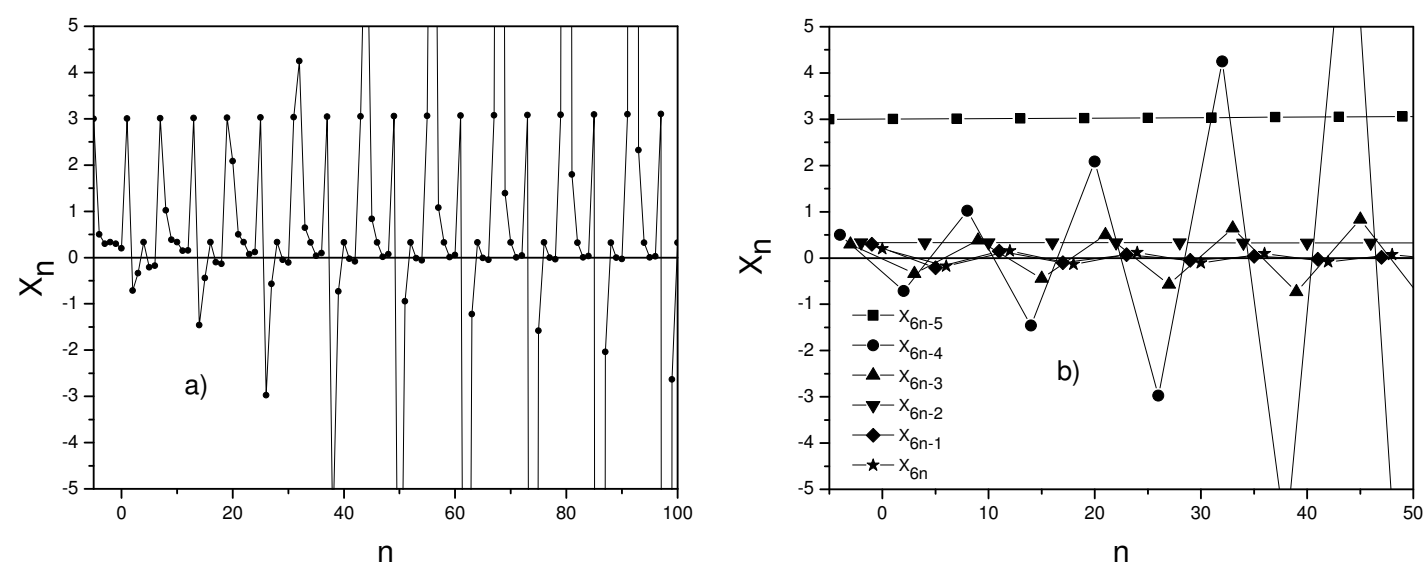

Figure 4: Using the parameter values $A=2, B=-4, C=-2$ and the initial data $a=0.2, b=0.3, c=\frac{1}{3}, d=0.3, e=0.5, f=3$; (a) the whole sequence $\left(x_{n}\right)_{n}$ is unbounded, (b) the corresponding subsequences are illustrated.

5. The case $C=-A, A B<0, c f=\frac{-2 A}{B}$, be $=\frac{-2 A}{B}, a d \in(-\infty, 0) \cup\left(\frac{-2 A}{B}, \infty\right)$, is investigated using the parameter values $A=2, B=-4, C=-2$ and the initial data $a=0.2, b=-3, c=0.25, d=-0.3$, $e=-\frac{1}{3}, f=4$. In Fig. 5 , we notice that $\left(x_{6 n-1}\right)_{n},\left(x_{6 n-2}\right)_{n},\left(x_{6 n-4}\right)_{n},\left(x_{6 n-5}\right)_{n}$ are constants (since $\left.x_{6 n-1}=b, x_{6 n-2}=c, x_{6 n-4}=e, x_{6 n-5}=f\right)$, the subsequence $\left(x_{6 n-3}\right)_{n}$ converges to zero, however the subsequence $\left(\left|x_{6 n}\right|\right)_{n}$ goes to infinity which is coherent to Theorem 5.4. Also, the whole solution is unbounded which is coherent to Theorem 5.7.
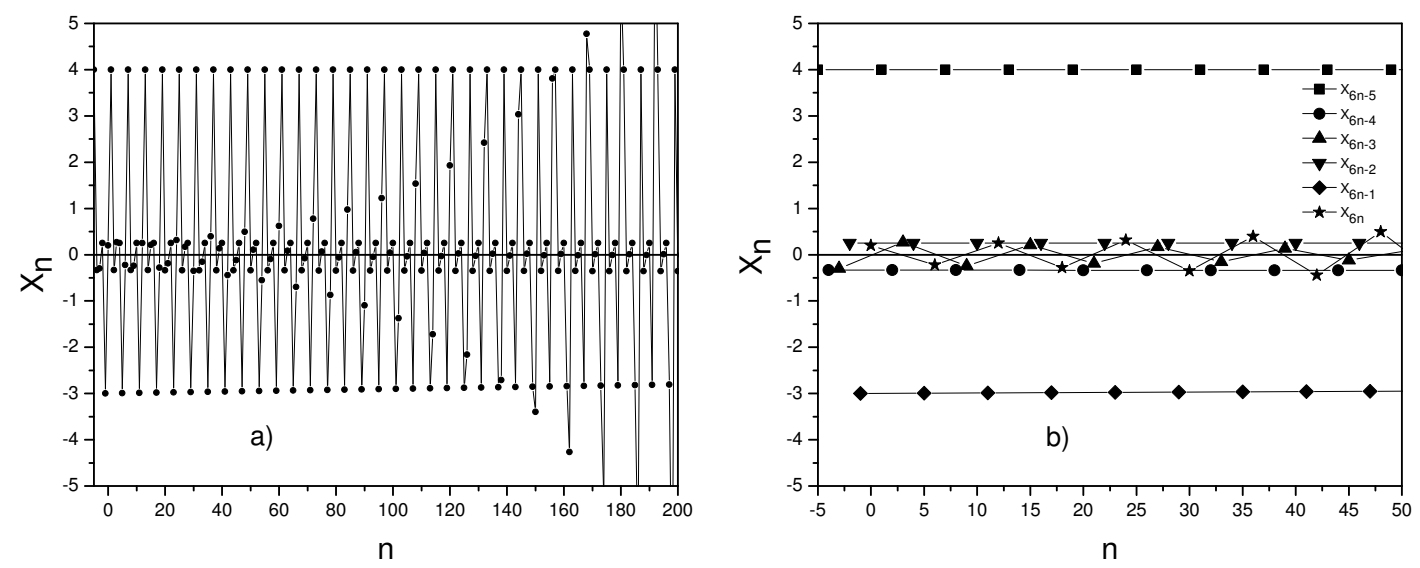

Figure 5: Using the parameter values $A=2, B=-4, C=-2$ and the initial data $a=0.2, b=-3, c=0.25, d=-0.3, e=-\frac{1}{3}$, $f=4 ;(a)$ the whole sequence $\left(x_{n}\right)_{n}$ is unbounded, (b) the corresponding subsequences are illustrated. 
6. The case $\left|\frac{A}{C}\right|>1,(A-C+B c f)(A-C+B b e)(A-C+B a d) \neq 0$, is studied using the parameter values $A=5, B=2, C=4$ and the initial data $a=2, b=-3, c=2, d=-2, e=-0.5, f=1$. In Fig. 6 , it is clear that the solution is damping to zero which is coherent to Theorem 5.6 part 2.
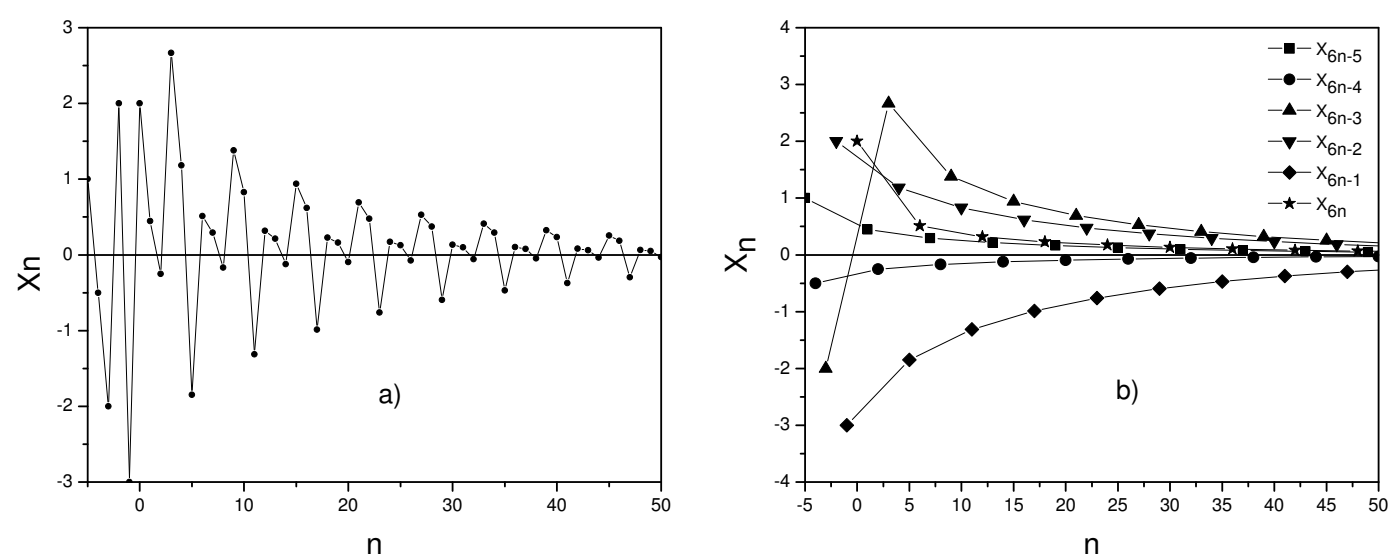

Figure 6: Using the parameter values $A=5, B=2, C=4$ and the initial data $a=2, b=-3, c=2, d=-2, e=-0.5, f=1 ;(a)$ the whole sequence $\left(x_{n}\right)_{n}$ converges to zero, $(b)$ the corresponding subsequences are illustrated.

7. The case $\left|\frac{A}{C}\right|>1,(A-C+B c f)(A-C+B b e) \neq 0, A-C+B a d=0$ is discussed using the parameter values $A=17, B=-3, C=5$ and the initial data $a=1, b=-2, c=2, d=4, e=-0.5, f=1$. In Fig. 7 , it is shown that the subsequences $\left(x_{6 n-1}\right)_{n},\left(x_{6 n-2}\right)_{n},\left(x_{6 n-4}\right)_{n},\left(x_{6 n-5}\right)_{n}$ converge to zero, and the subsequences $\left(x_{6 n-3}\right)_{n},\left(x_{6 n}\right)_{n}$ are constants $\left(\right.$ since $x_{6 n-3}=d$ and $\left.x_{6 n}=a\right)$. Thus, the whole sequence is bounded.
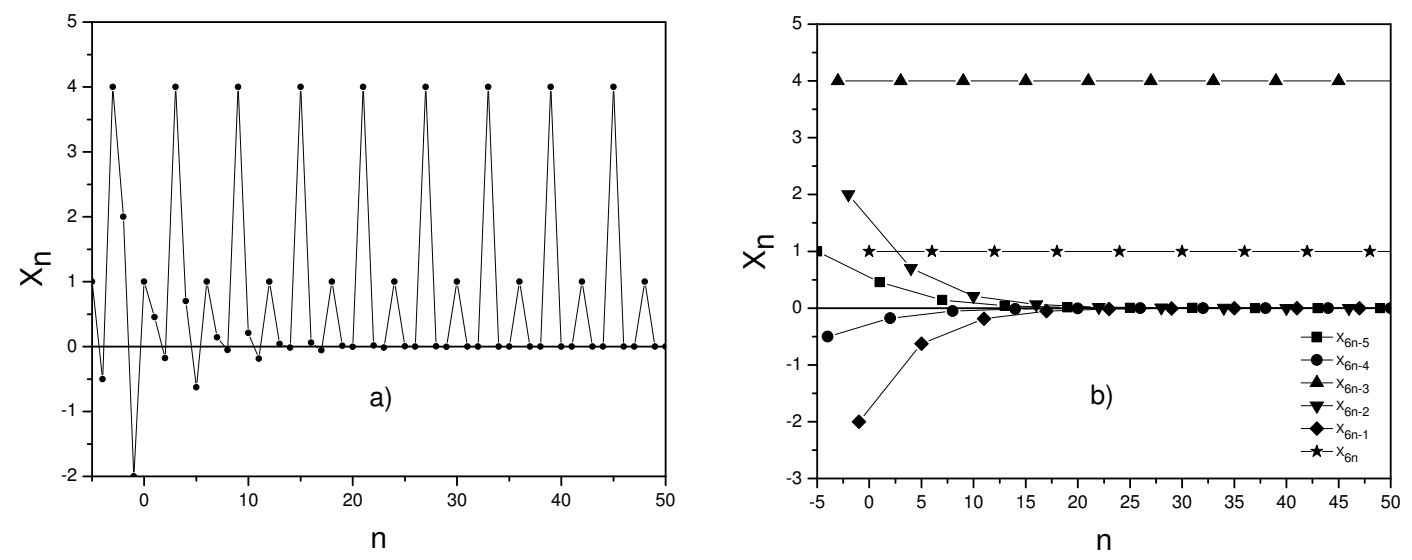

Figure 7: Using the parameter values $A=17, B=-3, C=5$ and the initial data $a=1, b=-2, c=2, d=4, e=-0.5, f=1 ;(a)$ the whole sequence $\left(x_{n}\right)_{n}$ is bounded, (b) the corresponding subsequences are illustrated.

8. The case $\left|\frac{A}{C}\right|>1, A-C+B c f \neq 0, A-C+B b e=A-C+B a d=0$ is illustrated in Fig. 8, with the parameter values $A=15, B=-1, C=3$ and the initial data $a=2, b=-3, c=1, d=6$, $e=-4, f=1$. In this figure it is shown that the subsequences $\left(x_{6 n-2}\right)_{n},\left(x_{6 n-5}\right)_{n}$ converge to zero and that the subsequences $\left(x_{6 n}\right)_{n},\left(x_{6 n-1}\right)_{n},\left(x_{6 n-3}\right)_{n},\left(x_{6 n-4}\right)_{n}$ are constants $\left(\right.$ since $x_{6 n}=a$, 
$x_{6 n-1}=b, x_{6 n-3}=d$ and $\left.x_{6 n-4}=e\right)$. So the whole sequence $\left(x_{n}\right)_{n}$ is bounded. This case is justified analytically in the proof of Theorem 5.6.
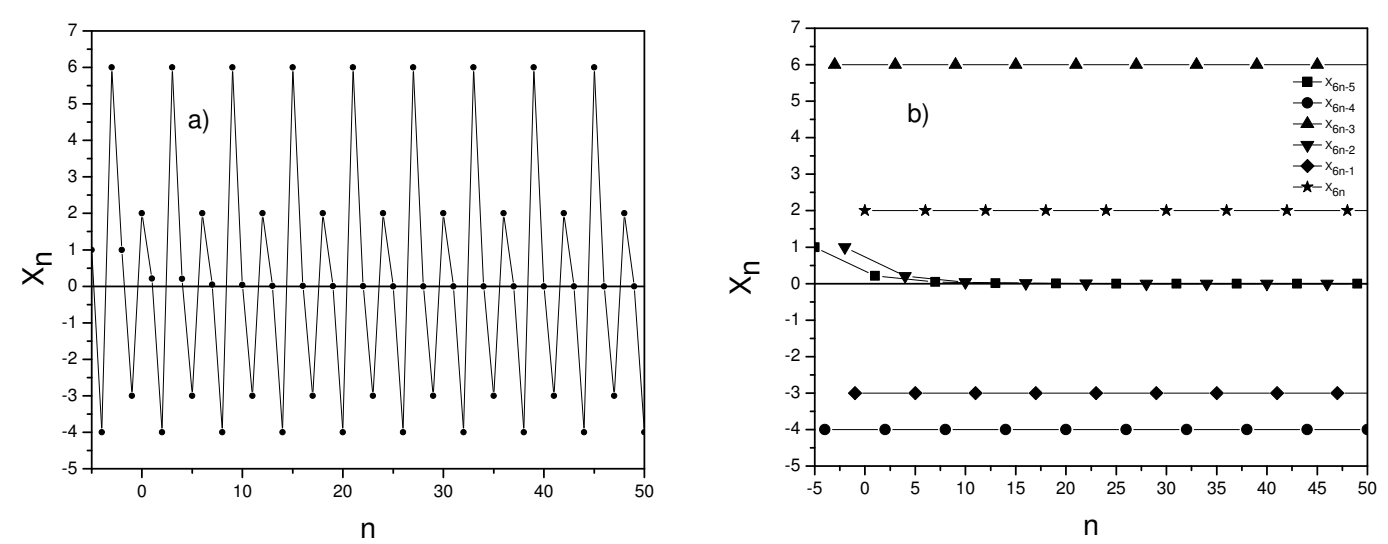

Figure 8: Using the parameter values $A=15, B=-1, C=3$ and the initial data $a=2, b=-3, c=1, d=6, e=-4, f=1 ;(a)$ the whole sequence $\left(x_{n}\right)_{n}$ is bounded, $(b)$ the corresponding subsequences are illustrated.

9. The case $\left|\frac{A}{C}\right|>1, A-C+B c f=A-C+B b e=A-C+B a d=0$ is illustrated in Fig. 9 in which we set the parameter values $A=9, B=-2, C=1$ and the initial data $a=1, b=-1, c=2, d=4$, $e=-4, f=2$. In this figure it is clear that the subsequences $\left(x_{6 n-j}\right)_{n}, j=0,1, \ldots, 5$ are constants (since $x_{6 n}=a, x_{6 n-1}=b, x_{6 n-2}=c, x_{6 n-3}=d, x_{6 n-4}=e, x_{6 n-5}=f$ ). This is justified analytically in the proof of Theorem 5.6. In Fig. 9, it is also depicted that the obtained solution is a periodic-6 solution

$$
f, e, d, c, b, a, f, e, d, c, b, a, \ldots
$$

This is in harmony with Theorem 6.2.
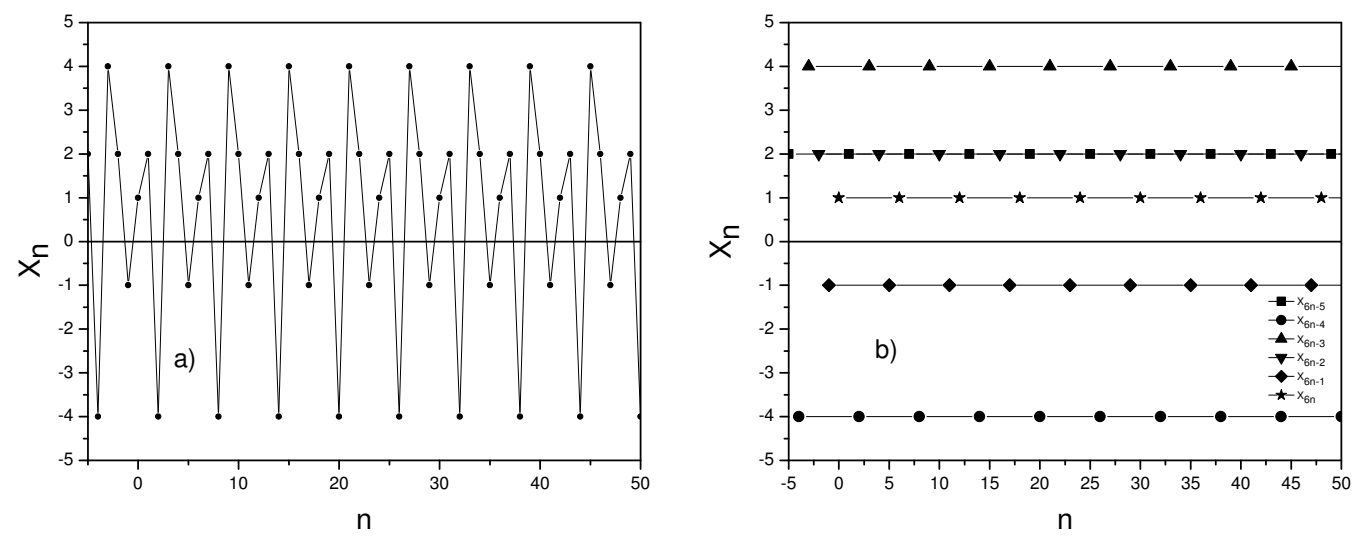

Figure 9: Using the parameter values $A=9, B=-2, C=1$ and the initial data $a=1, b=-1, c=2, d=4, e=-4, f=2 ;$ (a) the whole sequence $\left(x_{n}\right)_{n}$ exhibits a periodic- 6 solution, $(b)$ the subsequences $\left(x_{6 n-j}\right)_{n}, j=0,1, \ldots, 5$ are all constants.

\section{Conclusion}

We have studied the dynamical behaviors of the rational difference equation $x_{n+1}=\frac{C x_{n-5}}{A+B x_{n-2} x_{n-5}}$ with arbitrary initial conditions, where $A, B$, and $C$ are arbitrary constants. We have also obtained its 
general solution and discussed its asymptotic behaviors. Moreover, we have investigated the existence of periodic solutions in the proposed difference equation. Numerical simulations have been used to verify the correctness of analytical results.

\section{Acknowledgment}

The authors thank the Deanship of Research at the University of Hail, Saudi Arabia, for funding this work under Grant no. 0150287.

\section{References}

[1] H. N. Agiza, A. A. Elsadany, Chaotic dynamics in nonlinear duopoly game with heterogeneous players, Appl. Math. Comput., 149 (2004), 843-860. 1

[2] E. Ahmed, A. A. Elsadany, T. Puu, On Bertrand duopoly game with differentiated goods, Appl. Math. Comput., 251 (2015), 169-179. 1

[3] E. Ahmed, A. S. Hegazi, On dynamical multi-team and signaling games, Appl. Math. Comput., 172 (2006), 524-530. 1

[4] A. M. Amleh, V. Kirk, G. Ladas, On the dynamics of $x_{n+1}=\frac{a+b x_{n-1}}{A+B x_{n-2}}$, Math. Sci. Res. Hot-Line, 5 (2001), 1-15. 1

[5] S. S. Askar, The impact of cost uncertainty on Cournot oligopoly game with concave demand function, Appl. Math. Comput., 232 (2014), 144-149. 1

[6] S. S. Askar, A. M. Alshamrani, K. Alnowibet, The arising of cooperation in Cournot duopoly games, Appl. Math. Comput., 273 (2016), 535-542. 1

[7] C. Cinar, On the difference equation $x_{n+1}=\frac{x_{n-1}}{-1+x_{n} x_{n-1}}$, Appl. Math. Comput., 158 (2004), 813-816.

[8] C. Cinar, On the positive solutions of the difference equation $x_{n+1}=\frac{a x_{n-1}}{1+b x_{n} x_{n-1}}$, Appl. Math. Comput., 156 (2004), 587-590. 1

[9] Q. Din, Qualitative nature of a discrete predator-prey system, Contemp. Methods Math. Phys. Gravit. (Online), 1 (2015), 27-42.

[10] E. M. Elabbasy, H. El-Metwally, E. M. Elsayed, Global attractivity and periodic character of a fractional difference equation of order three, Yokohama Math. J., 53 (2007), 89-100. 1

[11] E. M. Elabbasy, A. A. Elsadany, Y. Zhang, Bifurcation analysis and chaos in a discrete reduced Lorenz system, Appl. Math. Comput., 228 (2014), 184-194. 1

[12] M. M. El-Dessoky, E. M. Elsayed, On the solutions and periodic nature of some systems of rational difference equations, J. Comput. Anal. Appl., 18 (2015), 206-218.

[13] H. El-Metwally, E. M. Elsayed, Form of solutions and periodicity for systems of difference equations, J. Comput. Anal. Appl., 15 (2013), 852-857.

[14] H. El-Metwally, E. M. Elsayed, Qualitative behavior of some rational difference equations, J. Comput. Anal. Appl., 20 (2016), 226-236.

[15] H. A. El-Morshedy, E. Liz, Convergence to equilibria in discrete population models, J. Difference Equ. Appl., 11 (2005), 117-131. 1

[16] H. A. El-Morshedy, E. Liz, Globally attracting fixed points in higher order discrete population models, J. Math. Biol., 53 (2006), 365-384.

[17] A. A. Elsadany, H. N. Agiza, E. M. Elabbasy, Complex dynamics and chaos control of heterogeneous quadropoly game, Appl. Math. Comput., 219 (2013), 11110-11118. 1

[18] A. A. Elsadany, A. E. Matouk, Dynamic Cournot duopoly game with delay, J. Complex Syst., 2014 (2014), 7 pages. 1

[19] E. M. Elsayed, Solution and attractivity for a rational recursive sequence, Discrete Dyn. Nat. Soc., 2011 (2011), 17 pages.

[20] E. M. Elsayed, Solutions of rational difference systems of order two, Math. Comput. Modelling, 55 (2012), 378-384. 1

[21] E. M. Elsayed, On the solutions and periodic nature of some systems of difference equations, Int. J. Biomath., 7 (2014), 26 pages. 1

[22] E. M. Elsayed, On a max type recursive sequence of order three, Miskolc Math. Notes, 17 (2016), 837-859.

[23] E. M. Elsayed, A. M. Ahmed, Dynamics of a three-dimensional systems of rational difference equations, Math. Methods Appl. Sci., 39 (2016), 1026-1038.

[24] E. M. Elsayed, A. Alghamdi, Dynamics and global stability of higher order nonlinear difference equation, J. Comput. Anal. Appl., 21 (2016), 493-503.

[25] E. M. Elsayed, T. F. Ibrahim, Periodicity and solutions for some systems of nonlinear rational difference equations, Hacet. J. Math. Stat., 44 (2015), 1361-1390. 
[26] E. M. Elsayed, T. F. Ibrahim, Solutions and periodicity of a rational recursive sequences of order five, Bull. Malays. Math. Sci. Soc., 38 (2015), 95-112.

[27] E. M. Elsayed, M. Mansour, M. M. El-Dessoky, Solutions of fractional systems of difference equations, Ars Combin., 110 (2013), 469-479.

[28] Y. Halim, Global character of systems of rational difference equations, Electron. J. Math. Anal. Appl., 3 (2015), $204-214$.

[29] S. S. Hassan, E. Chatterjee, Dynamics of the equation $z_{n+1}=\frac{\alpha+\beta z_{n}}{A+z_{n-1}}$ in the complex plane, Cogent Math., 2 (2015), 12 pages.

[30] D. Jana, E. M. Elsayed, Interplay between strong Allee effect, harvesting and hydra effect of a single population discrete-time system, Int. J. Biomath., 9 (2016), 25 pages.

[31] R. Karatas, C. Cinar, D. Simsek, On positive solutions of the difference equation $x_{n+1}=\frac{x_{n-5}}{1+x_{n-2} x_{n-5}}$, Int. J. Contemp. Math. Sci., 1 (2006), 494-500.

[32] A. Q. Khan, Q. Din, M. N. Qureshi, T. F. Ibrahim, Global behavior of an anti-competitive system of fourth-order rational difference equations, Comput. Ecol. Softw., 4 (2014), 35-46.

[33] V. L. Kocić, G. Ladas, Global behavior of nonlinear difference equations of higher order with applications, Mathematics and its Applications, Kluwer Academic Publishers Group, Dordrecht, (1993). 2.2

[34] A. E. Matouk, A. A. Elsadany, E. Ahmed, H. N. Agiza, Dynamical behavior of fractional-order Hastings-Powell food chain model and its discretization, Commun. Nonlinear Sci. Numer. Simul., 27 (2015), 153-167. 1

[35] D. Tollu, Y. Yazlik, N. Taskara, The solutions of four Riccati difference equations associated with Fibonacci numbers, Balkan J. Math., 2 (2014), 163-172.

[36] N. Touafek, E. M. Elsayed, On a second order rational systems of difference equations, Hokkaido Math. J., 44 (2015), 29-45.

[37] Y. Yazlik, E. M. Elsayed, N. Taskara, On the behaviour of the solutions of difference equation systems, J. Comput. Anal. Appl., 16 (2014), 932-941.

[38] D. Zhang, J. Huang, L.-Y. Wang, W.-Q. Ji, Global behavior of a nonlinear difference equation with applications, Open J. Discrete Math., 2 (2012), 78-81.

[39] Q. Zhang, W. Zhang, J. Liu, Y. Shao, On a fuzzy logistic dierence equation, WSEAS Trans. Math., 13 (2014), $282-290$. 1 